Published in final edited form as:

Melanoma Res. 2016 February ; 26(1): 35-41. doi:10.1097/CMR.0000000000000201.

\title{
Roles of adjuvant and salvage radiotherapy for desmoplastic melanoma
}

\author{
Daniel E. Olivera,b, Kirtesh R. Patel ${ }^{b}$, Jeffrey Switchenko ${ }^{e}$, Douglas Parker ${ }^{\dagger}$, David H. \\ Lawson $^{\mathrm{c}}$, Keith A. Delman ${ }^{\mathrm{d}}$, Ragini R. Kudchadkarc, and Mohammad K. Khan ${ }^{\mathrm{b}}$ \\ aEmory University School of Medicine, Winship Cancer Institute, Emory University, Atlanta, \\ Georgia, USA \\ bDepartment of Radiation Oncology, Winship Cancer Institute, Emory University, Atlanta, Georgia, \\ USA \\ 'Department of Hematology and Medical Oncology, Winship Cancer Institute, Emory University, \\ Atlanta, Georgia, USA \\ dDepartment of Surgical Oncology, Winship Cancer Institute, Emory University, Atlanta, Georgia, \\ USA \\ eDepartment of Biostatistics and Bioinformatics, Rollins School of Public Health, Emory \\ University, Atlanta, Georgia, USA \\ fDepartment of Surgical Pathology and Dermatology, Emory University, Atlanta, Georgia, USA
}

\section{Abstract}

Current guidelines are unclear as to the precise role of radiotherapy (RT) in patients with desmoplastic melanoma (DM). The purpose of this study was to evaluate our institutional outcomes in patients with DM, and to explore the roles of both adjuvant and salvage RT in these patients. We identified 100 patients with a histopathologic diagnosis of DM who received treatment at our institution from 2000 to 2014. Local control, distant metastasis-free survival, and overall survival (OS) were evaluated in the 95 patients managed surgically with or without adjuvant and/or salvage RT. The overall rate of local recurrence (LR) was 10\%. There was no LR in either adjuvant or salvage RT cohort. Adjuvant RT did not significantly improve LR-free survival at 5 years ( 100 vs. $81 \%, P=0.59$ ), despite the RTpatients having worse pathological features. Four of seven (57\%) salvage patients developed distant metastases, despite $100 \%$ local control. Adjuvant RT did not significantly impact 5 -year overall survival ( 86 vs. $82 \%, P=0.43$ ). RT shows a trend towards improved local control in both the adjuvant and salvage settings for patients with DM, and likely overcomes adverse risk factors after surgery in appropriately selected patients. Future prospective studies are needed to better address the optimal management for these patients.

Correspondence to Mohammad K. Khan, MD, PhD, Department of Radiation Oncology, Winship Cancer Institute, Emory University, 1365 Clifton Road NE, Room A-1312, Atlanta, GA 30322, USA, Tel: + 1404778 4126; fax: + 1404778 4126;

drkhurram2000@gmail.com.

Conflicts of interest: There are no conflicts of interest. 


\section{Keywords}

control; desmoplastic; local recurrence; melanoma; prognostic factors; radiation; radiotherapy; regional; risk factors; salvage

\section{Introduction}

Desmoplastic melanoma (DM), a rare variant of melanoma, was first described in 1971 by Conley et al. [1] as a variant of spindle-cell melanoma with a fibroblastic (desmoplastic), collagenous stroma. Patients with DM frequently experience delays in diagnosis, with more advanced lesions on presentation than in conventional melanoma. This is likely due to an amelanotic, scar-like clinical appearance. There is a wide histopathologic differential for DM, including spindle-cell sarcoma, neurofibroma, schwannoma, blue nevus, sarcomatoid squamous cell carcinoma, and scar [2-6]. One of the defining characteristics of DM is an increased propensity for local recurrence (LR), with rates reported from 4 to $60 \%[2,4,6-$ 11], compared with less than $5 \%$ in other melanomas [12]. Several studies $[6,13,14]$ have shown DM to be associated with perineural invasion (PNI); this characteristic, in addition to increased Breslow thickness, advanced Clark level, and a predilection for the head and neck region, all likely play roles in the increased tendency towards LR $[6,11,14-16]$. Due to the fibromatous nature of DM, as well as the inherent challenges of head and neck surgery, the extent of surgical margins following initial resection has frequently come into question [2,11, 17-19]. Despite increased rates of LR, DM patients have been shown to develop less locoregional and distant spread, when compared with other melanomas of a similar tumor stage $[4,20]$.

Current guidelines are unclear about the precise role of radiotherapy (RT) in the treatment of DM, stating that RT may be 'considered' for selected DM patients with narrow margins [21]. Among practices nationally, there is substantial variation in terms of which patients are to be selected for RT, as well as when radiation should be given, either as initial adjuvant therapy or later in the salvage modality. There are also conflicting reports about the potential role of RT in improving patient outcomes when other adverse pathological risks factors (PNI, extent of desmoplasia, positive margins, or recurrent disease) are also present $[2,11,14-17]$. It has been argued that surgery-alone, with strict adherence to adequate margins, is sufficient for preventing LR in DM [2]. However, a pair of recently published studies has provided further clarification on the role of RT, reinforcing the association between adjuvant RT and improved local control $[15,16]$. There has, however, been little published that compares the efficacy of adjuvant to salvage radiation therapy. Thus, in our study, we further explore these roles, while adding additional patient outcomes to the existing literature in support of RT.

\section{Methods}

Following IRB approval, the charts of over 2200 melanoma patients receiving surgical treatment at our institution between the years of 2000 and 2014 were reviewed, and all patients with a histopathologically confirmed diagnosis of DM were identified. Medical records were reviewed for demographic information, tumor characteristics, and treatment 
characteristics. Additionally, RT databases were reviewed to ensure that no DM patients were missed. Investigators of this study worked closely with the dermatopathologist (D.P.) to ensure clarity of diagnosis and tumor characteristics. All pathology reports pertaining to biopsy and tumor resection were reviewed. We identified 100 patients with DM, with or without PNI (Table 1). One patient presenting with DM in the setting of a previous non-DM at the same primary site was excluded, as were four patients presenting for palliative therapy. These patients were included for descriptive purposes only, and the review was limited to 95 DM patients. Whenever data were available, patients were subdivided into those with pure DM (pDM) and mixed DM (mDM), based on the criteria described in 2004 by Busam et al. [13], in which tumors with more than $90 \%$ desmoplasia were defined as pDM, and those between 10 and $90 \%$ were defined as $\mathrm{mDM}$. For the mDM patients, the subtype of non-DM was recorded. Results of immunohistochemistry, such as S-100, melan-A and HMB-45 were recorded where data were available.

All patients either received wide local excision at our institution, or had been referred from an outside facility for management following surgery. Patients were analyzed in accordance with American Joint Commission on Cancer (AJCC) criteria, and all patients presented with stages I-III (any T with or without ulceration, any N, M0), and none had distant metastases on presentation. The majority (87\%) of patients received a sentinel lymph node biopsy (SLNB), and all patients with evidence of either clinical or microscopic lymph node (LN) disease underwent lymphadenectomy.

The primary outcome measure in this study was local control, defined as freedom from LR. LR was defined as development of DM within the initial primary site after surgery or RT. Adjuvant RT was defined as being given after initial surgery. Salvage RT was defined as either postoperative salvage RT or inoperable salvage RT; the term 'salvage' refers to RT of a recurrence which occurred after initial surgical failure, while the term 'postoperative' refers to a repeat surgery due to the recurrence, and 'inoperable' refers to no further repeat surgery. In all adjuvant and postoperative salvage cases, RT was given within 3 months of surgery. Locoregional recurrence was defined as either in-transit metastasis (any LR occurring $>2 \mathrm{~cm}$. from the primary lesion between the primary and the draining LN basin), or recurrence of the disease within the area encompassing draining lymphatics and regional nodal basin. Distant metastasis was defined as metastasis to any distant nodal, visceral or cutaneous regions. Additional outcomes of interest were distant metastasis-free survival (DMFS) and overall survival (OS).

\section{Statistical methods}

Three treatment comparisons were analyzed: (a) adjuvant versus no radiation therapy/ salvage (nonadjuvant), (b) no radiation therapy versus salvage, and (c) adjuvant versus salvage. Time-to-event endpoints included LR-free survival, DMFS, and OS; local control was defined as the absence of LR. Three patients did not have follow-up date information, and were thus excluded from the survival analysis (censoring only took place if a patient had a last follow-up date). Descriptive statistics were generated for each of the three treatment groups. 
For the first treatment comparison, local control was defined as the absence of LR from date of diagnosis to last documented follow-up date. For the no RT cohort in the second treatment comparison, local control was defined in a similar fashion as above; for the salvage therapy cohort, local control was defined as the absence of LR from first day of salvage radiation treatment to last documented follow-up date. Thus, we compare the overall control rate for the no RT cohort versus the further control rate for the salvage therapy cohort with the second treatment comparison. A local control analysis was not possible for the third treatment comparison, since there were no cases that had a LR after RT in the adjuvant and the salvage settings.

For all three comparisons, DMFS was defined as time from date of diagnosis to date of distant metastasis or last documented follow-up date and/or date of death. Similarly, OS was defined as time from date of diagnosis to either date of last documented follow-up date and/or death.

Univariate (UV) Cox proportional hazards models were fit for all comparisons and endpoints, where applicable. Multivariate (MV) Cox models were fit for OS and DMFS for the adjuvant versus nonadjuvant comparison, and for the no radiation therapy versus salvage comparison. Covariates that were statistically significant in the UV models were included with treatment in the MV models. Proportional hazards assumptions were checked. In the adjuvant versus nonadjuvant comparison, there were no LRs in the adjuvant cohort. As a result, local recurrence-free survival curves were estimated using the Kaplan-Meier method, but a log-rank test was not performed and Cox models were not fit. Fisher's exact tests were used to compare the local control rates between the adjuvant and nonadjuvant treatment cohorts, as well as the no radiation therapy and salvage cohorts. OS and DMFS curves were estimated using the Kaplan-Meier method, and cohorts and covariates were compared using $\log$-rank tests. The statistical analysis was performed using SAS 9.3 (SAS Institute Inc., Cary, North Carolina, USA), and Kaplan-Meier curves were generated using R 2.15.1 (Statistics Department, University of Auckland, New Zealand). Significance was assessed at the 0.05 level.

\section{Radiotherapy}

All patients undergoing RT received treatment at our institution, except for one patient whose RT details at an outside facility were unavailable (Table 2). Of those patients receiving RT, 10 received true adjuvant RT following initial local excision, seven received postoperative salvage RT, and four received palliative RT. Patients receiving palliative RT alone were treated for severe pain and/or bleeding; they were included in the study for descriptive purposes, but excluded from any comparisons in treatment outcomes. These patients received palliative doses with intensity-modulated RT; 20-30 Gy total, using 3-6 Gy per fraction within 20 days. The targeted RT region for adjuvant and salvage patients included the surgical site plus a 1-4 cm margin. Other RT characteristics are presented in Table 1. Response of the tumor to salvage RT was evaluated clinically in all patients, and with additional radiographs in the other six (two by MRI alone, one by PET computed tomography alone, two by MRI/PET computed tomography, one by chest radiography). 


\section{Results}

\section{Patient and tumor characteristics}

We identified 100 total patients with DM, nearly $4.4 \%$ of more than 2200 melanoma patients initially screened. The median age at diagnosis was 62.4 years (range, 27-92 years). There were 72 men and 28 women, a 2.6:1 ratio. Three patients were without a last documented follow-up date. The primary site of DM in these patients was most commonly the head and neck (40\%), followed by trunk (24\%), upper extremity (22\%), with the remaining being either lower extremity or acral lesions. Median Breslow thickness was $2.75 \mathrm{~mm}$ (range, $0.65-18 \mathrm{~mm}$ ), with a mean of $4.6 \mathrm{~mm}$. Clark level data was available for 98 of 100 patients, with nearly all showing advanced levels of invasion: $95 \%$ had either Clark level IV or V tumors, while the remaining $3 \%$ had either Clark levels II or III tumors. While we attempted to record proximity of tumor to surgical margins on all patients, $71 \%$ of the patients that had 'clear' margins that were not further quantified. Other details of the patients treated and tumor characteristics are shown in Table 1.

In those patients (90 of 100) for whom LNs were successfully assessed and staged on presentation, $80(89 \%)$ were found to have N0 disease (Table 1). Six (6.7\%) presented with $\mathrm{N} 1$ disease, three (3.3\%) presented with N2 disease, and only one (1.1\%) presented with N3 disease. All but one of the patients with evidence of LN disease had received an SLNB; this patient received excision at an outside facility for a $1 \mathrm{~mm}$ scalp lesion with microscopically positive margins and no other risk factors. The patient was then referred after several weeks for a clinically palpable and disease positive cervical node, for which he underwent reexcision of the primary site as well as a cervical lymphadenectomy, in which three of 43 nodes were positive. All six of the N1 patients were diagnosed with nodal disease by SLNB. One of the patients with $\mathrm{N} 2$ disease was found to have intradermal in-transit metastasis but disease was absent in zero of 16 LNs. All patients with evidence of nodal disease on presentation received a lymphadenectomy, with two of nine patients showing further nodal disease: one patient was described previously, with six of 17 nodes being positive in the other patient. Both received subsequent radiation in the form of photons to the nodal basin. All patients presenting with nodal disease were of the mDM subtype. No patients were found to have distant metastases on presentation.

\section{Local recurrence and predicting factors}

The overall rate of LR in this series was $10 \%$. In the 85 patients managed initially with surgery-alone, 10 went on to develop LR (11.8\%), seven of these would go on to receive salvage RT after one or more recurrences. In the 10 patients who received adjuvant postoperative radiation, there were no LRs with $100 \%$ local control (Fig. 1). We found no significant difference in local control rates $(P=0.59)$. Individual characteristics and disease outcomes of patients receiving RT in our study are shown in Table 2. In addition to the adjuvant RT group, all patients receiving salvage RT experienced 100\% local control, despite having had failed surgical management previously. All six salvage patients with imaging showed clinical and radiographic evidence of tumor regression. 
Due to the absence of LR following RT in the RT cohorts, no significant comparisons could be made regarding predictors for LR. The mean initial Breslow thickness for the 10 patients with LR was $6.4 \mathrm{~mm}$ (range, $1.7-18 \mathrm{~mm}$ ). Nine (90\%) of these patients had at least a Clark level IV tumor. The remaining patient had a Clark level III tumor. Five (50\%) patients had an initial DM in the head and neck region. PNI was found to be present in three patients (30\%), with another patient's PNI status denoted as 'indeterminate'. Three (30\%) patients were found to have pDM subtype. Regarding surgical margins, of the 10 patients who had LR, two had microscopically positive margins, three had margins less than or equal to $1 \mathrm{~mm}$, and the other five had 'clear' margins that were not quantified.

\section{Distant metastasis}

In total, 10 of the 95 (11\%) patients developed distant metastasis. Five of 78 (6.4\%) patients managed with surgery-alone developed distant metastasis, one of $10(10 \%)$ patients receiving adjuvant RT developed distant metastasis, and four of seven (57\%) patients receiving salvage RT went on to develop distant metastasis (Table 2). On comparison of nonadjuvant RT (surgery-alone/salvage RT) versus adjuvant RT, only absence of ulceration was found to be significantly associated with improved DMFS on both UV [hazard ratio (HR), 0.24; 95\% CI, 0.60-0.96 ( $P=0.028)$ ] and MV [HR, 0.24; 95\% CI, 0.06-0.96 ( $P=$ $0.044)]$ analysis. In comparing surgery-alone versus postoperative salvage RT, the surgeryalone cohort was strongly associated with improved DMFS [HR, 0.14; 95\% CI, 0.037-0.524 $(P<0.001)$ ] suggesting a more favorable cohort in the surgery-alone group. Absence of ulceration also showed a trend towards improved DMFS on UV analysis in this comparison as well [HR, $0.30 ; 95 \% \mathrm{CI}, 0.07-1.36(P=0.09)]$.

\section{Overall survival}

Table 3 shows UV and MV OS. As shown in Fig. 2, OS at 5 years did not differ significantly based on receipt of adjuvant RT (86 vs. 82\%) [HR, 0.55; 95\% CI, 0.12-2.49 $(P=0.43)$ ]. Factors significantly associated with increased OS on UV analysis were absence of ulceration [HR, 0.23 ; 95\% CI, 0.08-0.69 $(P=0.004)]$ and absence of lymphovascular space invasion [HR, 0.19; 95\% CI, 0.04-0.85 ( $P=0.015)]$. On MV analysis, absence of ulceration was significantly associated with increased OS [HR, 0.23; 95\% CI, 0.08-0.71 $(P=0.011)$, while absence of lymphovascular space invasion showed a trend towards increased OS [HR, $0.21 ; 95 \% \mathrm{CI}, 0.04-1.08(P=0.062)$ ]. Two patients receiving adjuvant RT died from progressive non-DM melanoma, while three of the seven patients receiving salvage RT died from progression of their DMM (Table 2).

\section{Discussion}

Our finding of DM patients at a rate of $4.4 \%$ of all cutaneous melanoma patients is in accordance with previous reports ( $<4 \%$ of melanoma cases) $[6,16,22,23]$. Additionally, it is possible that the challenges inherent in diagnosis may have caused a number of DM patients to be overlooked. The median Breslow thickness of $2.75 \mathrm{~mm}$ in this study was less than has been recorded in other reports $[2,11,14-17]$. This may account for a lower overall LR in our surgical cohort than in other studies $[4,6,11,15,16,23]$. 
A 2008 Sydney Melanoma Unit study suggested that adjuvant RT might offset some of the unfavorable risk factors predicting for LR in DM [14]. In their study, LR rates were comparable between patients managed with surgery-alone and those receiving adjuvant RT; this was despite worse disease (thicker Breslow, more advanced Clark level, narrower excision margins) in the latter cohort. In our study, there were similar differences in patient groups (Table 1). The patients receiving adjuvant RT had thicker tumors than those managed with surgery-alone (mean Breslow of 10.5 vs. $3.5 \mathrm{~mm}$ ). Their tumors were also more likely to be Clark level V ( 90 vs. 19\%), head and neck lesions (50 vs. 37\%), ulcerated (30 vs. $16 \%$ ), and associated with PNI (70 vs. 24\%). Additionally, the adjuvant RT cohort had more patients with the pDM subtype (70 vs. $28 \%$ ), which has recently been associated with risk factors for LR such as increased Breslow thickness, advanced Clark level, and PNI [24]. Despite worse features in our radiation cohort, our local control was $100 \%$. In looking at a combined 184 total DM patients receiving postoperative adjuvant RT from two recent studies, local control was $93 \%$ in each study.

Despite a substantial sample of DM patients, the sizes of the adjuvant (10 patients) and salvage (seven patients) RT cohorts did not allow for further statistical testing. However, it is encouraging that there were no further LRs in either of these cohorts. Regarding the salvage RT cohort specifically, it is of note that these patients also achieved excellent local control despite high-risk features and poor DMFS outcomes. In light of the 2013 data from MD Anderson and Moffitt, these patients would have been ideal for selection to adjuvant RT due to these prognostic features $[20,21]$.

This idea was illustrated by one of the salvage patients in Table 2 . The patient presented with a $17 \mathrm{~mm}$ DM lesion in his thigh; the excised tumor was found to be a Clark level V, with positive margins. Three LRs developed over the following 27 months despite multiple attempts at surgical salvage, at which point he was treated with a salvage regimen of radiation to the primary site, $55.2 \mathrm{~Gy}$ in 23 fractions. Despite ongoing disease progression (lumbar spine, liver, pulmonary, and posterior pharynx metastases), this patient experienced no further LRs after RT before dying 19 months later. All patients receiving RT, even in the salvage cohort, had surgery before radiation. Since the salvage patients had recurrence following initial surgery to begin with, it was thought that the likelihood of success with a salvage surgery-alone was probably even less; thus RT was offered in this case. Additionally, these patients often had narrow/positive margins in a head and neck site, where surgeryalone may have been insufficient.

Limitations of this study include the potential for selection bias and referral for adjuvant/ salvage RT based on physician preference, the retrospective design, the heterogeneous delivery of RT, and the differing baseline characteristics of the patients involved in the study. The inability to conduct thorough comparisons between treatment cohorts due to the small numbers in the RT cohorts is another limitation of this study; larger patient cohorts would have allowed more impactful UV and MV analyses.

\section{Conclusion}

Recent retrospective data suggests that adjuvant RT is effective in reducing LR for high-risk DM patients $[15,16]$. Despite the limited number of patients receiving RT in our study, our 
research supports the recent literature by suggesting that adjuvant RT improves local control in patients with adverse features. In addition, by evaluating those patients receiving salvage RT separately, we show that the absence of LR in the setting of poor survival and higher rates of distant metastasis is a compelling argument for consideration of upfront adjuvant RT for these patients. Prospective randomized trials with larger cohorts will only serve to solidify the role of RT in the primary management of DM. NCT00975520 is a trial (phase 3 at this time) comparing surgery-alone with surgery plus postoperative radiation in patients with widely resected neurotropic melanoma of the head and neck; this should provide valuable data for future decision-making. However, there is now already considerable evidence for a more specific selection criteria.

\section{Acknowledgments}

Research reported in this publication was supported in part by the Biostatistics and Bioinformatics Shared Resource of Winship Cancer Institute of Emory University and NIH/NCI under award number P30CA138292. The content is solely the responsibility of the authors and does not necessarily represent the official views of the National Institutes of Health.

\section{References}

1. Conley J, Lattes R, Orr W. Desmoplastic malignant melanoma (a rare variant of spindle cell melanoma). Cancer. 1971; 28:914-936. [PubMed: 5286448]

2. Arora A, Lowe L, Su L, Rees R, Bradford C, Cimmino VC, et al. Wide excision without radiation for desmoplastic melanoma. Cancer. 2005; 104:1462-1467. [PubMed: 16080180]

3. Feng Z, Wu X, Chen V, Velie E, Zhang Z. Incidence and survival of desmoplastic melanoma in the United States, 1992-2007. J Cutan Pathol. 2011; 38:616-624. [PubMed: 21518379]

4. Lens MB, Newton-Bishop JA, Boon AP. Desmoplastic malignant melanoma: a systematic review. Br J Dermatol. 2005; 152:673-678. [PubMed: 15840097]

5. McCarthy SW, Scolyer RA, Palmer AA. Desmoplastic melanoma: a diagnostic trap for the unwary. Pathology. 2004; 36:445-451. [PubMed: 15370114]

6. Quinn MJ, Crotty KA, Thompson JF, Coates AS, O'Brien CJ, McCarthy WH. Desmoplastic and desmoplastic neurotropic melanoma: experience with 280 patients. Cancer. 1998; 83:1128-1135. [PubMed: 9740077]

7. Carlson JA, Dickersin GR, Sober AJ, Barnhill RL. Desmoplastic neurotropic melanoma. A clinicopathologic analysis of 28 cases. Cancer. 1995; 75:478-494. [PubMed: 7812919]

8. Jaroszewski DE, Pockaj BA, DiCaudo DJ, Bite U. The clinical behavior of desmoplastic melanoma. Am J Surg. 2001; 182:590-595. [PubMed: 11839322]

9. Reiman HM, Goellner JR, Woods JE, Mixter RC. Desmoplastic melanoma of the head and neck. Cancer. 1987; 60:2269-2274. [PubMed: 3440236]

10. Smithers BM, McLeod GR, Little JH. Desmoplastic melanoma: patterns of recurrence. World J Surg. 1992; 16:186-190. [PubMed: 1373257]

11. Vongtama R, Safa A, Gallardo D, Calcaterra T, Juillard G. Efficacy of radiation therapy in the local control of desmoplastic malignant melanoma. Head Neck. 2003; 25:423-428. [PubMed: 12784232]

12. Tsao H, Atkins MB, Sober AJ. Management of cutaneous melanoma. N Engl J Med. 2004; 351:998-1012. [PubMed: 15342808]

13. Busam KJ, Mujumdar U, Hummer AJ, Nobrega J, Hawkins WG, Coit DG, Brady MS. Cutaneous desmoplastic melanoma: reappraisal of morphologic heterogeneity and prognostic factors. Am J Surg Pathol. 2004; 28:1518-1525. [PubMed: 15489657]

14. Chen JY, Hruby G, Scolyer RA, Murali R, Hong A, Fitzgerald P, et al. Desmoplastic neurotropic melanoma: a clinicopathologic analysis of 128 cases. Cancer. 2008; 113:2770-2778. [PubMed: 18823042] 
15. Guadagnolo BA, Prieto V, Weber R, Ross MI, Zagars GK. The role of adjuvant radiotherapy in the local management of desmoplastic melanoma. Cancer. 2014; 120:1361-1368. [PubMed: 24142803]

16. Strom T, Caudell JJ, Han D, Zager JS, Yu D, Cruse CW, et al. Radiotherapy influences local control in patients with desmoplastic melanoma. Cancer. 2014; 120:1369-1378. [PubMed: 24142775]

17. Foote MC, Burmeister B, Burmeister E, Bayley G, Smithers BM. Desmoplastic melanoma: the role of radiotherapy in improving local control. ANZ J Surg. 2008; 78:273-276. [PubMed: 18366400]

18. Skelton HG, Smith KJ, Laskin WB, McCarthy WF, Gagnier JM, Graham JH, Lupton GP. Desmoplastic malignant melanoma. J Am Acad Dermatol. 1995; 32(Pt 1):717-725. [PubMed: 7722014]

19. Smithers BM, McLeod GR, Little JH. Desmoplastic, neural transforming and neurotropic melanoma: a review of 45 cases. Aust N Z J Surg. 1990; 60:967-972. [PubMed: 2268214]

20. Hawkins WG, Busam KJ, Ben-Porat L, Panageas KS, Coit DG, Gyorki DE, et al. Desmoplastic melanoma: a pathologically and clinically distinct form of cutaneous melanoma. Ann Surg Oncol. 2005; 12:207-213. [PubMed: 15827812]

21. Coit DG, Thompson JA, Andtbacka R, Anker CJ, Bichakjian CK, Carson WE 3rd, et al. National Comprehensive Cancer Network. Melanoma, version 4.2014. J Natl Compr Canc Netw. 2014; 12:621-629. [PubMed: 24812131]

22. Chen LL, Jaimes N, Barker CA, Busam KJ, Marghoob AA. Desmoplastic melanoma: a review. J Am Acad Dermatol. 2013; 68:825-833. [PubMed: 23267722]

23. Wasif N, Gray RJ, Pockaj BA. Desmoplastic melanoma - the step-child in the melanoma family? J Surg Oncol. 2011; 103:158-162. [PubMed: 21259250]

24. Murali R, Shaw HM, Lai K, McCarthy SW, Quinn MJ, Stretch JR, et al. Prognostic factors in cutaneous desmoplastic melanoma: a study of 252 patients. Cancer. 2010; 116:4130-4138.

[PubMed: 20564101] 


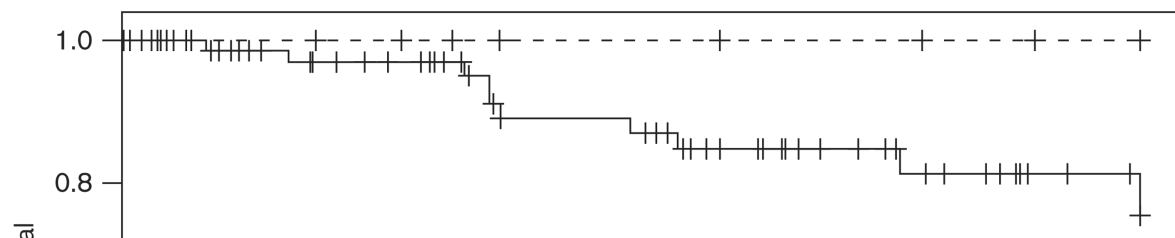

$\sum_{\frac{\pi}{2}}^{\frac{\pi}{2}}$

๑ $0.6-$

d

$\frac{2}{2}$

$0.4-$

임

0.2

0.0

5-years LRFS: $81 \%$ (nonadjuvant) vs. $100 \%$ (adjuvant), $P=0.59-$ Nonadjuvant

0

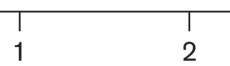

3
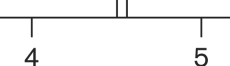

Time (years)

Number at risk

\begin{tabular}{|c|c|c|c|c|c|c|}
\hline Nonadjuvant 82 & 61 & 51 & 42 & 28 & 21 & 14 \\
\hline Adjuvant 10 & 8 & 5 & 4 & 3 & 2 & 1 \\
\hline
\end{tabular}

Fig. 1.

Local recurrence-free survival (LRFS) for postoperative adjuvant radiotherapy cohort compared with nonadjuvant cohorts. 


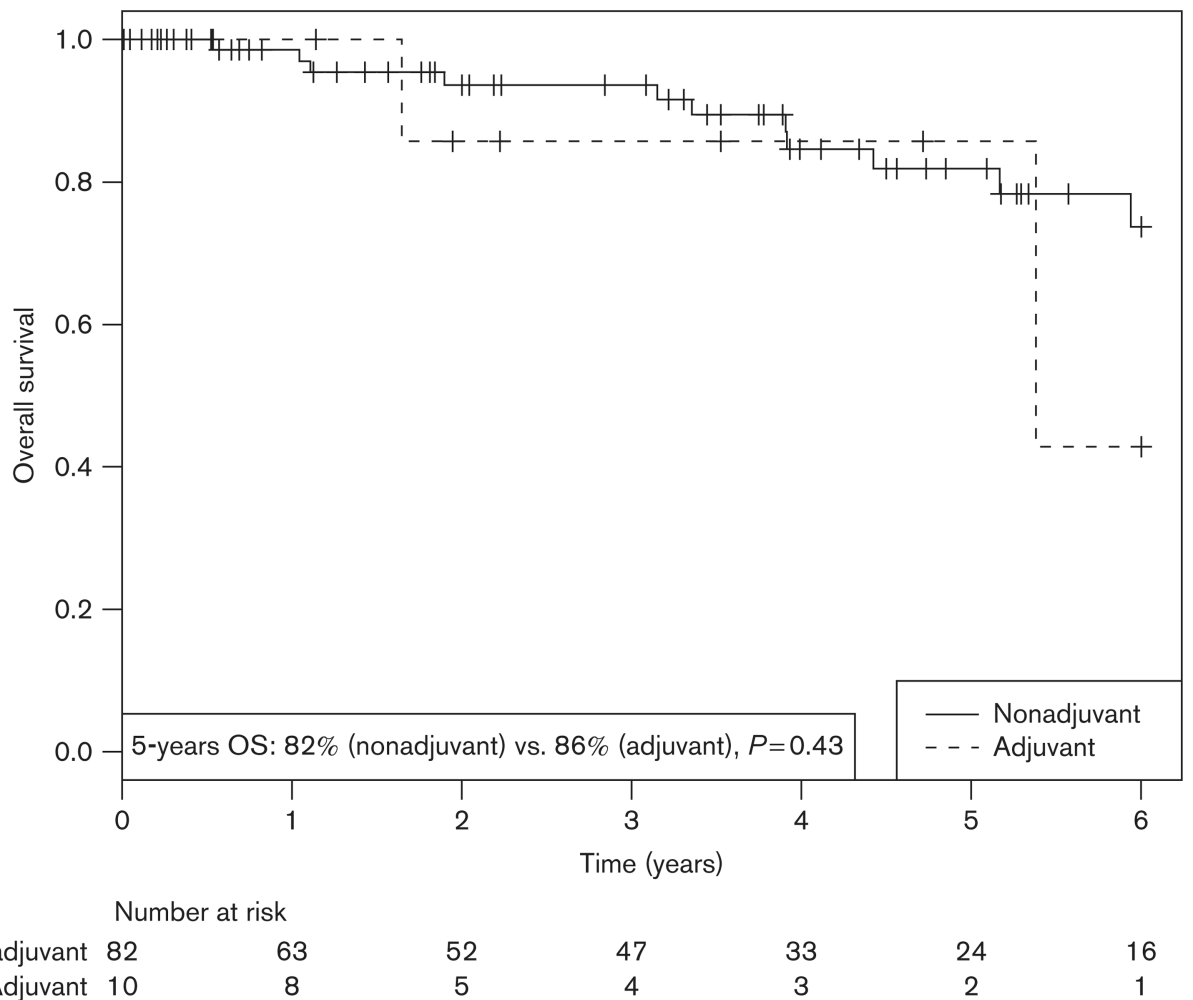

Fig. 2.

Overall survival (OS) for postoperative adjuvant radiotherapy cohort compared with nonadjuvant cohorts. 
Table 1

Patient and treatment characteristics

\begin{tabular}{|c|c|c|c|c|}
\hline \multirow[b]{2}{*}{ Characteristics } & \multicolumn{3}{|c|}{$[n(\%)]$} & \multirow[b]{2}{*}{$\boldsymbol{P}$} \\
\hline & No RT $(N=78)$ & Adjuvant RT $(N=10)$ & Salvage RT $(N=7)$ & \\
\hline Mean age \pm SD (years) & $61.3 \pm 12.3$ & $57.9 \pm 15.9$ & $63.4 \pm 16.8$ & 0.657 \\
\hline Median age (range) (years) & $62(27-85)$ & $60(28-78)$ & $62(43-92)$ & \\
\hline \multicolumn{5}{|l|}{ Sex } \\
\hline Male & $55(70)$ & $8(80)$ & $4(57)$ & 0.623 \\
\hline Female & $23(30)$ & $2(20)$ & $3(43)$ & \\
\hline \multicolumn{5}{|l|}{ Site of primary lesion } \\
\hline Head and neck & $29(37)$ & $5(50)$ & $2(33)$ & 0.862 \\
\hline Trunk & $21(27)$ & $1(10)$ & $1(17)$ & \\
\hline Upper extremity & $17(22)$ & $3(30)$ & $2(33)$ & \\
\hline Lower extremity & $6(8)$ & $1(10)$ & $1(17)$ & \\
\hline Acral & $5(6)$ & - & - & \\
\hline Mean Breslow thickness $\pm \mathrm{SD}(\mathrm{mm})$ & $3.5 \pm 2.8$ & $10.5 \pm 4.7$ & $8.2 \pm 6.6$ & $<0.001$ \\
\hline Median Breslow thickness (range) $(\mathrm{mm})$ & $2.5(0.6-17)$ & $10.8(3.8-16.3)$ & $6.1(2.8-18)$ & \\
\hline \multicolumn{5}{|l|}{ Clark level } \\
\hline $\mathrm{V}$ & $15(19)$ & $9(90)$ & $6(86)$ & $<0.001$ \\
\hline $\mathrm{IV} / \mathrm{V}$ & $6(8)$ & - & - & \\
\hline IV & $53(69)$ & $1(10)$ & $1(14)$ & \\
\hline II/III & $3(4)$ & - & - & \\
\hline \multicolumn{5}{|l|}{ Histology } \\
\hline Pure & $22(28)$ & $7(70)$ & $2(29)$ & 0.030 \\
\hline Mixed & $56(72)$ & $3(30)$ & $5(71)$ & \\
\hline \multicolumn{5}{|l|}{ Ulceration } \\
\hline Absent & $64(84)$ & $7(70)$ & $4(67)$ & 0.251 \\
\hline Present & $12(16)$ & $3(30)$ & $2(33)$ & \\
\hline \multicolumn{5}{|l|}{ PNI } \\
\hline Absent & $59(76)$ & $3(30)$ & $3(50)$ & 0.004 \\
\hline Present & $19(24)$ & $7(70)$ & $3(50)$ & \\
\hline \multicolumn{5}{|l|}{ LVSI } \\
\hline Absent & $77(99)$ & $9(90)$ & $4(67)$ & 0.008 \\
\hline Present & $1(1)$ & $1(10)$ & $2(33)$ & \\
\hline \multicolumn{5}{|l|}{ LNs at diagnosis } \\
\hline Yes & $5(7)$ & $2(29)$ & $1(20)$ & 0.079 \\
\hline No & $69(93)$ & $5(71)$ & $4(80)$ & \\
\hline \multicolumn{5}{|l|}{ Excision margins } \\
\hline Clear but not quantified & $64(82)$ & $2(20)$ & $3(43)$ & $<0.001$ \\
\hline$<1 \mathrm{~mm}$ & $4(5)$ & $5(50)$ & $2(29)$ & \\
\hline $1.1-5 \mathrm{~mm}$ & $3(4)$ & - & - & \\
\hline$>1 \mathrm{~cm}$ & $1(1)$ & $1(10)$ & $1(14)$ & \\
\hline
\end{tabular}




\begin{tabular}{|c|c|c|c|c|}
\hline \multirow[b]{2}{*}{ Characteristics } & \multicolumn{3}{|c|}{$[n(\%)]$} & \multirow[b]{2}{*}{$P$} \\
\hline & No RT $(N=78)$ & Adjuvant RT $(N=10)$ & Salvage RT $(N=7)$ & \\
\hline Positive & $6(8)$ & $2(20)$ & $1(14)$ & \\
\hline \multicolumn{5}{|c|}{ RT total dose (Gy) } \\
\hline 30 & - & $4(40)$ & $3(43)$ & - \\
\hline 48 & - & $3(30)$ & - & \\
\hline $50-66$ & - & $3(30)$ & $3(43)$ & \\
\hline Unknown & - & - & $1(14)$ & \\
\hline \multicolumn{5}{|l|}{ RT fractions } \\
\hline 5 & - & $4(40)$ & $3(43)$ & - \\
\hline $20-33$ & - & $6(60)$ & $3(43)$ & \\
\hline Unknown & - & - & $1(14)$ & \\
\hline \multicolumn{5}{|l|}{ RT technique } \\
\hline Electrons & - & $4(40)$ & - & - \\
\hline Photons & - & $6(60)$ & $5(71)$ & \\
\hline Unknown & - & - & $2(29)$ & \\
\hline \multicolumn{5}{|l|}{ LN RT } \\
\hline Yes & - & $2(20)$ & $2(29)$ & - \\
\hline No & - & $8(80)$ & $4(57)$ & \\
\hline Unknown & - & - & $1(14)$ & \\
\hline
\end{tabular}

Palliative radiotherapy cohort not included in this table.

LN, lymph node; LVSI, lymphovascular space invasion; PNI, perineural invasion; RT, radiotherapy. 


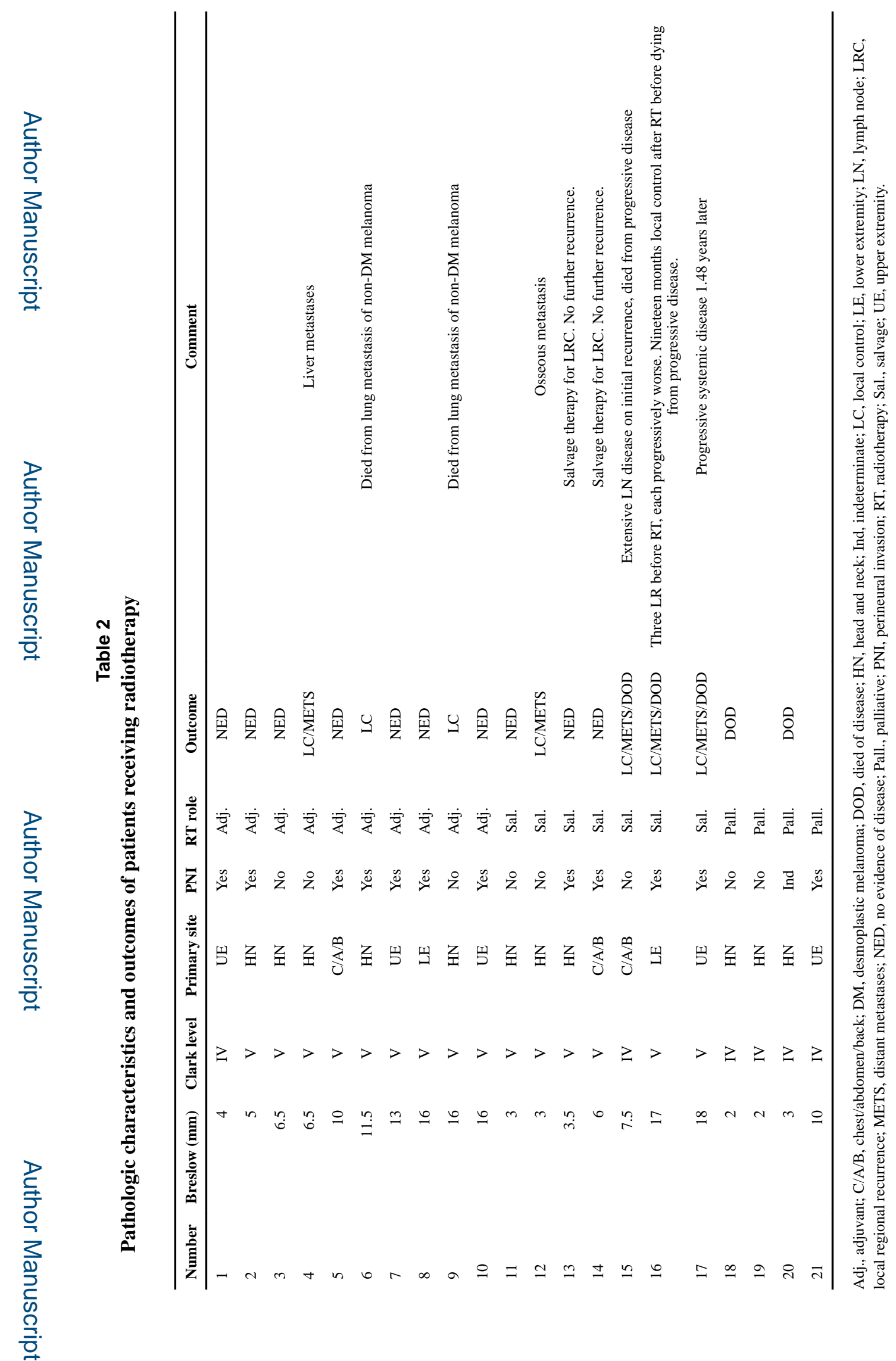

Melanoma Res. Author manuscript; available in PMC 2016 May 17. 


\section{를 \\ 골}

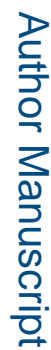



Melanoma Res. Author manuscript; available in PMC 2016 May 17. 